\title{
VARIATIONAL ASPECTS OF THE SEIBERG-WITTEN FUNCTIONAL
}

\author{
Jürgen Jost, Xiaowei Peng and Guofang Wang
}

\section{INTRODUCTION}

Recently, Seiberg and Witten (see [SW1], [SW2] and [W]) introduced a new monopole equation which yields new differential-topological invariants of four dimensional manifolds, closely related to the Donaldson polynomial invarints [DK]. This equation has been used to give more elementary proof of many heorems in gauge theory and to obtain many new results (see [KM], [Le], [T1], [T2] and [T4]).

These equations are first order equations, but the solutions also satisfy more general second order equations, in a similar way as (anti) self dual connections are solutions of the Yang-Mills equations or holomorphic maps between Kähler manifolds are harmonic. Sometimes, one can use index theorems or methods from algebraic geometry to construct solutions or to get information about the space of solutions. Another strategy would be to first solve the second order equations and then try to identify conditions under which certain solutions of the second order equations actually also solve the first order ones. This strategy has been successful in many other instances. One of the reasons for this success usually was the variational structures of the second order equations, namely, one could identify them as the Euler-Lagrange equations of some variational integral. The solutions of the first order equations are absolute minimum of this functional.

We believe that this strategy should also be usefully for the Seiberg-Witten equations. We therefore study the corresponding variational integral which we call Seiberg-Witten functional. We show that weak solutions of the Euler-Lagrange equations are smooth. The main result of our paper is that the functional satisfies the Palais-Smale condition. As a consquence, one not only obtains the existence of the minimizers (among which one hopes to find solutions of the first order equations), but also the existence of unstable solutions. More precisely, one knows how to deduce from the Palais-Smale conditon the mountain pass lemma and a general Ljusternik-Schnirelmann type theory, and even Morse (-Bott) theory if the functiional is a Morse (-Bott) functional. We hope that these additional non-minimizing solutions (which do not solve the first order equations) also carry useful geometric information and can be used to define more invariants for differential 4-manifolds. This will be studied elsewhere, however.

When we had already nearly completed this paper, the questions that are answered here were also posed by P. Braam at a conference at the ICTP in Trieste.

1991 Mathematics Subject Classification. 58E15, 53C07.

Key words and phrases. Seiberg-Witten equation, Seiberg-Witten functional, regularity and Palais-Smale condition. 
This paper was written while the third auther was a guest of the research project SFB 237 of the DFG at the Ruhr University Bochum. The first auther was also supported by the DFG.

\section{The Seiberg-Witten equation}

In order to be able to write down the equation of Seiberg-Witten, we need to recall the definition of a $S_{p i n}{ }^{c}$-structure. (For details see [LM]).

Let $(X, g)$ be an oriented, compact Riemannian 4-manifold and $P_{S O(4)} \rightarrow X$ its oriented orthonormal frame bundle. $\operatorname{Spin}^{c}(4)=\operatorname{Spin}(4) \times_{\mathbb{Z}_{2}} U(1)$. A $\operatorname{Spin}^{c}$ structure is a lift of the structure group $\mathrm{SO}(4)$ to $\operatorname{Spin}^{c}(4)$, i.e. there exists a principal $S$ pin ${ }^{c}$-bundle $P_{S p i n^{c}(4)} \rightarrow X$ such that there is a bundle map

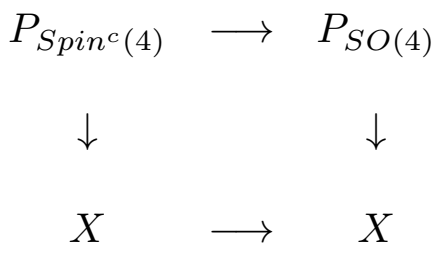

Let $Q=P_{\operatorname{Spin}^{c}(4)} / \operatorname{Spin}(4)$ be a principal $U(1)$-bundle. $W=P_{\operatorname{Spin}^{c}(4)} \times_{\operatorname{Spin}^{c}(4)}$ $\mathbb{C}^{4}$ and $L=Q \times_{U(1)} \mathbb{C}$ resp. is the associated spinor bundle and the line bundle resp.. $W$ can be decomposed globally as $W^{+}$and $W^{-}$. Locally,

$$
W^{ \pm}=S^{ \pm} \otimes L^{1 / 2}
$$

Here $S^{ \pm}$is a spinor bundle w. r. t. a local Spin-struture on $X$. Both $S^{ \pm}$and $L^{1 / 1}$ are locally defined.

There exists a Clifford multiplication

$$
T X \times W^{+} \rightarrow W^{-}
$$

denoted by $e \cdot \phi \in W^{-}$for $e \in T X$ and $\phi \in W^{+}$. Here $T X$ is the tangent bundle of $X$. A connection on the bundle $W^{+}$can be defined by the Levi-Civita connection and a connection on $L$. The "twisted" Dirac operator $D_{A}: \Gamma\left(W^{+}\right) \rightarrow \Gamma\left(W^{-}\right)$is defined by

$$
D_{A}=\sum_{i=1}^{4} e_{i} \cdot \nabla_{A} .
$$

Here, $\Gamma\left(W^{ \pm}\right)$is the space of sections of $W^{ \pm},\left\{e_{i}\right\}$ is an orthonormal basis of $T X$ and $\nabla_{A}$ is a connection on $W^{+}$induced by the Levi-Civita connection and a connection $A$ on the line bundle $L$.

Definition 1.1. The Seiberg-Witten equations are

$$
\begin{aligned}
D_{A} \phi & =0, \\
F_{A}^{+} & =\frac{i}{4}\left\langle e_{i} e_{j} \phi, \phi\right\rangle e^{i} \wedge e^{j},
\end{aligned}
$$

for $A$ a connection on $L$ and $\phi \in \Gamma\left(W^{+}\right)$, where $F(A)=-i F_{A}$ is the curvature of $A, F_{A}^{+}$is the self dual part of $F_{A}$ and $\left\{e^{i}\right\}$ is the dual basis of $\left\{e_{i}\right\}$. 
Definition 1.2. The Seiberg-Witten functional is

$$
S W(A, \phi)=\int_{X}\left(\left|D_{A}\right|^{2}+\left|F_{A}^{+}-\frac{i}{4}\left\langle e_{i} e_{j} \phi, \phi\right\rangle e^{i} \wedge e^{j}\right|^{2}\right) d v o l
$$

The Euler-Lagrange equations of the Seiberg-Witten functional are

$$
\begin{aligned}
D_{A}^{*} D_{A} \phi-\frac{i}{2} F_{A}^{+} \cdot \phi-\frac{1}{8}\left\langle e_{i} e_{j} \phi, \phi\right\rangle e_{i} e_{j} \phi & =0, \\
d^{*}\left(F_{A}^{+}-\frac{i}{4}\left\langle e_{i} e_{j} \phi, \phi\right\rangle e^{i} \wedge e^{j}\right)+\frac{1}{2} \operatorname{Im}\left\langle D_{A} \phi, e_{i} \phi\right\rangle e^{i} & =0 .
\end{aligned}
$$

Here, $D_{A}^{*}$ is the formal adjoint operator of $D_{A}, d^{*}=-* d *$ and $*$ is the Hodge star operator.

It is easy to see that a solution of (1.1) is a solution of (1.3). In fact, it is clear from the definition of the functional that a solution of (1.1) is a minimizer of the Seiberg-Witten equation.

The following Weitzenböck formula plays an important role in the Seiberg-Witten theory,

$$
D_{A}^{*} D_{A} \phi=-\Delta_{A} \phi+\frac{s}{4} \phi+\frac{i}{2} F_{A} \cdot \phi
$$

where $s$ is the scalar curvature of $(X, g)$. By this formula, the Seiberg-Witten functional can be rewritten as

$$
S W(A, \phi)=\int_{X}\left(\left|\nabla_{A} \phi\right|^{2}+\left|F_{A}^{+}\right|^{2}+\frac{s}{4}|\phi|^{2}+\frac{1}{8}|\phi|^{4}\right) d v o l .
$$

And the Euler-Lagrange equation (1.3) can be rewritten as

$$
\begin{aligned}
-\Delta_{A} \phi+\frac{s}{4} \phi+\frac{1}{4}|\phi|^{2} \phi & =0, \\
d^{*} F_{A}^{+}+\frac{1}{2} \operatorname{Im}\left\langle\nabla_{i} \phi, \phi\right\rangle e^{i} & =0 .
\end{aligned}
$$

Here $\Delta_{A}$ is the analyst's Laplacian, the negative Laplacian, and $\nabla_{i}=\nabla_{e_{i}}$.

Lemma 1.3. For a smooth solution $(A, \phi)$ of equation (1.3) (or (1.6)), $|\phi|(x) \leq$ $\max \left\{-s_{0}, 0\right\}$, where $s_{0}=\min \{s(x) \mid x \in X\}$.

Proof. From the maximum principle.

Corollary 1.4. If the scalar curvature of $X$ is nonnegative, then for any smooth solution $(A, \phi)$ of (1.2), $\phi \equiv 0$.

Hence, a solution of (1.2) shares many properties with solutions of the SeibergWitten equation (1.1). In section 3 below, we shall prove Lemma 1.3 for weak solutions of (1.2).

In this paper we shall consider the Seiberg-Witten functional and prove a compactness theorem. The precise set-up of the problem will be given in section 2 .

Before ending the present section, we want to give the definition of the Palais- 
Let $M$ be a Banach manifold and $f: M \rightarrow \mathbb{R}$ a smooth functional. Let $G$ be a Lie group acting on $M$ and suppose $f$ is invariant under $G$, i.e. for any $g \in G$ and $x \in X, f(g x)=f(x) . \quad f$ is said to satisfy the Palais-Smale condition: if for any sequence $\left\{x_{i}\right\}_{i \in \mathbb{N}}$ with

(i) $f\left(x_{i}\right)$ is bounded,

(ii) $d f\left(x_{i}\right) \rightarrow 0$, as $i \rightarrow \infty$,

there exists a subsequence (also denoted by $\left\{x_{i}\right\}$ ) and a sequence $g_{i} \in G$ such that $g_{i} x_{i}$ converges in $X$ to a critical point $x$ of $f$, i.e. $d f(x)=0$, with $f(x)=$ $\lim _{i \rightarrow \infty} f\left(x_{i}\right)$.

\section{THE SET-UP}

We need to choose a suitable working space to discuss the Seiberg-Witten functional.

For a vector bundle with a metric over $X$, we define $L^{k, p}(E)$, the Sobolev space of sections of $E$ by completing the space $\Gamma(E)$ of smooth sections of $E$ by

$$
\|s\|_{L^{k, p}}=\left(\sum_{|\alpha|=0}^{k} \int\left|\nabla^{\alpha} s\right|^{p}\right)^{1 / p},
$$

where $\nabla$ is a fixed metric connection on $E$. (For details see $[\mathrm{P}]$ ). For such Sobolev spaces, the Sobolev embedding theorem and the Hölder inequality are valid, i.e.

$$
\left(\int_{X}|s|^{4}\right)^{1 / 4} \leq c\|s\|_{L^{1.2}}, \quad \text { for } s \in L^{1,2},
$$

and

$$
\left(\int_{X}\left|s_{1}\right|^{2}\left|s_{2}\right|^{2}\right)^{1 / 2} \leq\left(\int_{X}\left|s_{1}\right|^{4}\right)^{1 / 4}\left(\int_{X}\left|s_{2}\right|^{4}\right)^{1 / 4} .
$$

Let $L^{1,2}\left(W^{+}\right)$be the space of sections of the bundle $W^{+}$of class $L^{1,2}$ defined above and $\mathcal{A}^{1,2}=L^{1,2}(\mathcal{A})$ the space of connections of class $L^{1,2}$ defined by a fixed connection $A_{0} . L^{1,2}\left(W^{+}\right)$and $\mathcal{A}^{1,2}$ are Hilbert manifolds.

Lemma 2.1. The Seiberg-Witten functional $S W$ is well defined on $\mathcal{A}^{1,2} \times L^{1,2}\left(W^{+}\right)$ and $S W$ is smooth.

Proof. From (2.1) and (2.2),

$$
\int_{X}|A \phi|^{2} \leq c\|A\|_{L^{1,2}}\|\phi\|_{L^{1,2}}
$$

This implies that $S W$ is well-defined. It is easy to check that $S W$ is smooth.

Now let us to choose a suitable Lie group as a gauge group. First let $\mathcal{G}_{0}=$ $\exp \left(i L^{2,2}(X, \mathbb{R})\right)$. We claim that $\mathcal{G}_{0}$ is a Lie group. Actually, $\mathcal{G}_{0}$ can be seen as a quotient of $L^{2,2}(X, \mathbb{R})$ under an equivalence relation $\sim \phi_{1} \sim \phi_{2}$ if and only if $\phi_{1}(x)-\phi_{2}(x)=2 \pi n$, for almost all $x \in X$, for some integer $n$. It is clear that $Y=L^{2,2} / \sim$ is a Lie group with the usual addition of functions. $\mathcal{G}_{0}$ can be identified with $Y$ by the exponential map. Hence $\mathcal{G}_{0}$ is a Lie group with the multiplication of functions, as the identity component in the continuous case. Then by a wellknown result about harmonic maps from $X$ into $S^{1}$ ([EL]), in any component of $C^{\infty}\left(X, S^{1}\right)$ there exists a unique map $g \in C^{\infty}\left(X, S^{1}\right)$ such that

$$
d^{*}\left(g^{-1} d g\right)=0 \text {, }
$$

$$
g\left(x_{0}\right)=1, \quad \text { for a fixed point } \quad x_{0} \in X
$$


Lemma 2.2. Let $\mathcal{G}=\cup g \cdot \mathcal{G}_{0}$. $\mathcal{G}$ is a Lie group, where the union is over all components of $C^{\infty}\left(X, S^{1}\right)$.

Proof. Since $\mathcal{G}_{0}$ is a Lie group, it suffices to check the following two points.

(i) For two different components of $C^{\infty}\left(X, S^{1}\right)$ with as $g_{1}$ and $g_{2}$ obtained above, $g_{1} \mathcal{G}_{0} \cap g_{2} \mathcal{G}_{0}=\emptyset$.

Assuming that $g_{1} \mathcal{G}_{0} \cap g_{2} \mathcal{G}_{0} \neq \emptyset$, there exist $\varphi_{1}$ and $\varphi_{2} \in \mathcal{G}_{0}$ such that $g_{1} \varphi_{1}=$ $g_{2} \varphi_{2}$, for almost all $x \in X$. From the definition of $\mathcal{G}_{0}$, there exist $\zeta_{1}$ and $\zeta_{2} \in$ $L^{2,2}(X, \mathbb{R})$ such that

$$
g_{1} g_{2}^{-1}=e^{i\left(\zeta_{2}-\zeta_{1}\right)},
$$

for almost all $x \in X$. From (2.3) above, we have

$$
d^{*} d\left(\zeta_{2}-\zeta_{1}\right)=0,
$$

i.e. $\zeta_{2}-\zeta_{1} \in L^{2,2}(X, \mathbb{R})$ is a harmonic function. Hence tegether with $(2.4), \zeta_{2}=\zeta_{1}$. Therefore $g_{1}=g_{2}$, a contradiction.

(ii) The operation of the group is closed.

Let $g_{1} \varphi_{1} \in g_{1} \mathcal{G}_{0}, g_{2} \varphi_{2} \in g_{2} \mathcal{G}_{0}$, then

$$
g_{1} \varphi_{1} \cdot g_{2} \varphi_{2}=g_{1} g_{2} \varphi_{1} \varphi_{2} \in g_{1} g_{2} \mathcal{G}_{0}
$$

and $g_{1} g_{2}$ satisfies (2.3) and (2.4) and is the corresponding $g$ of some component of $C^{\infty}(X, R)$ as above.

Lemma 2.3. $\mathcal{G}$ acts smoothly on $\mathcal{A}^{1,2} \times L^{1,2}\left(W^{+}\right)$.

Proof. $\mathcal{G}$ acts on $\mathcal{A}^{1,2} \times L^{1,2}\left(W^{+}\right)$as follows

$$
g(A, \phi)=\left(g(A), g^{-1} \phi\right)
$$

for $(A, \phi) \in \mathcal{A}^{1,2} \times L^{1,2}\left(W^{+}\right)$and $g \in \mathcal{G}$, where $g(A)=A+g^{-1} d g$. It is easy to check that the action is well-defined and smooth.

Remark 2.4. In the non-Abelian case, an element $g$ of the gauge group of class $L^{2,2}$ need not act smoothly on $\mathcal{A}^{1,2}$.

So on $\mathcal{A}^{1,2} \times L^{1,2}\left(W^{+}\right)$, we can consider the Seiberg-Witten functional. It is easy to check that (1.2) is equivalent to (1.5) by an approximation argument. Here, we prefer to use the form (1.5).

Lemma 2.5. The Seiberg-Witten functional $S W$ is coercive, i.e., there exists a constant $c>0$ such that for each $(A, \phi) \in \mathcal{A}^{1,2} \times L^{1,2}\left(W^{+}\right)$

$$
S W(A, \phi) \geq c^{-1}\left(\left\|g^{-1} \phi\right\|_{L^{1,2}}+\|g(A)\|_{L^{1,2}}\right)-c
$$

for some $g \in \mathcal{G}$.

Proof. First, we have

$$
\begin{aligned}
S W(A, \phi) & =\int\left(\left|\nabla_{A} \phi\right|^{2}+\left|F_{A}^{+}\right|^{2}+\frac{s}{4}|\phi|^{2}+\frac{1}{8}|\phi|^{4}\right) \\
& =\int\left\{\left(\left|\nabla_{A} \phi\right|^{2}\right)+\frac{1}{16}|\phi|^{4}\right. \\
& \left.+\frac{1}{16}\left(4 s^{2}+4 s|\phi|^{2}+|\phi|^{4}\right)-\frac{1}{4} s^{2}\right\} \\
& >\int\left(\left|\nabla_{A} \phi\right|^{2}+\left|F_{A}^{+}\right|^{2}+\frac{1}{}|\phi|^{4}\right)-c .
\end{aligned}
$$


Using the Hölder inequality, we have

$$
\left(\int|\phi|^{2}\right)^{1 / 2} \leq c\left(\int|\phi|^{4}\right)^{1 / 4} \leq c \int|\phi|^{4}+c .
$$

In this paper, $c$ is a constant that may change from term to term.

Also, we have

$$
\begin{aligned}
\int_{X}|\nabla \phi|^{2} & \leq \int\left|\nabla_{A} \phi-\left(A-A_{0}\right) \phi\right|^{2} \\
& \leq 2 \int\left(\left|\nabla_{A} \phi\right|^{2}+\left|A-A_{0}\right|^{2}|\phi|^{2}\right) \\
& \leq 2 \int\left|\nabla_{A} \phi\right|^{2}+2\left(\int\left|A-A_{0}\right|^{4}\right)^{1 / 2}\left(\int|\phi|^{4}\right)^{1 / 2}
\end{aligned}
$$

Together with estimate (2.10) below, it follows that

$$
\int|\nabla \phi|^{2} \leq c \int\left|\nabla_{A} \phi\right|^{2}+c \int\left|F_{A}\right|^{2}+c
$$

Now we esitmate the term containing $g(A)$. There exists a standard method for the Abelian case. For convenience, we give a complete proof.

Step 1 (gauge fixing). There exists $g_{0} \in \mathcal{G}_{0}$ such that

$$
d^{*}\left(g_{0}(A)-A_{0}\right)=0
$$

Since $g_{0}=e^{i \zeta}$ for some $\zeta \in L^{2,2}(X, \mathbb{R}),(2.7)$ is equivalent to

$$
d^{*} d \zeta=i d^{*}\left(A-A_{0}\right)
$$

(2.8) is solvable, for $\int_{X} d^{*}\left(A-A_{0}\right)=0$ and $A \in L^{1,2}$. By the elliptic estimate, we have

$$
c^{-1}\left(\|A\|_{L^{1,2}}+\|\phi\|_{L^{1,2}}\right) \leq\|g(A)\|_{L^{1,2}}+\left\|g^{-1} \phi\right\|_{L^{1,2}} \leq c\left(\|A\|_{L^{1,2}}+\|\phi\|_{L^{1,2}}\right),
$$

for some constant $c$. Hence for simplicity, we denote $g(A)$ by $A$. So $d^{*}\left(A-A_{0}\right)=0$.

Step 2 (component fixing). The component group of $C^{\infty}\left(X, S^{1}\right)$ is isomorphic to $H^{1}(X, \mathbb{Z})$. For any component of $C^{\infty}\left(X, S^{1}\right)$, there exists a map $g$ satisfying $(2.3)$ and (2.4). We know

$$
g(A)-A_{0}=g^{-1} d g+A-A_{0} .
$$

The harmonic part of $g(A)-A_{0}$ is the harmonic part of $A-A_{0}$ plus the harmonic part of $g^{-1} d g$. Since the harmonic part of $g^{-1} d g$ belongs to $H^{1}(X, \mathbb{Z})$ and the Jacobi torus $H^{1}(X, \mathbb{R}) / H^{1}(X, \mathbb{Z})$ is compact, we can choose a component such that the harmonic part of $g(A)-A_{0}$ is bounded. Since $g$ is harmonic, $d^{*}\left(g(A)-A_{0}\right)=0$.

Step 3. Using the Hodge decomposition, we have 
where $H\left(g(A)-A_{0}\right)$ is the harmonic part of $g(A)-A_{0}$. From the preceding discussion, we obtain

$$
\begin{aligned}
\left\|g(A)-A_{0}\right\|_{L^{1,2}} & \leq c\left\|d\left(g(A)-A_{0}\right)\right\|_{L^{2}}+c \\
& \leq c\|d(g(A))\|_{L^{2}}+c \\
& \leq c\left\|F_{A}\right\|_{L^{2}}+c .
\end{aligned}
$$

Therefore,

$$
\left\|g(A)-A_{0}\right\|_{L^{1,2}} \leq c\left\|F_{A}\right\|_{L^{2}}+c \leq c\left\|F_{A}^{+}\right\|_{L^{2}}+c
$$

the last inequality is from that $\left\|F_{A}^{+}\right\|_{L^{2}}^{2}-\left\|F_{A}^{-}\right\|_{L^{2}}^{2}$ is independent of $A$. Now (2.6) and (2.10) imply

$$
S W(A, \phi) \geq c^{-1}\left(\left\|g^{-1} \phi\right\|_{L^{1,2}}+\|g(A)\|_{L^{1,2}}\right)-c .
$$

\section{Regularity of WEAK SOLUtions}

As in the Yang-Mills case, for the Seiberg-Witten equations there is some kind of removing singularity theorem (see [Z] and for Yang-Mills see [FU] and [U]). Actually, we shall prove in this section that all weak solutions of the second order equations (1.3) are smooth. This result will be used in the proof of the Main Theorem.

Theorem 3.1. Let $(A, \phi) \in \mathcal{A}^{1,2} \times L^{1,2}\left(W^{+}\right)$be a weak solutin of $(1.3)$, i.e. $(A, \phi)$ is a critical point of the Seiberg-Witten functional. Then there exists a gauge transfomation $g \in \mathcal{G}$ such that $g(A, \phi)=\left(g(A), g^{-1} \phi\right)$ is smooth.

First, we show the boundedness of $\|\phi\|_{L^{\infty}}$ for a weak solution.

Lemma 3.2. Let $(A, \phi) \in \mathcal{A}^{1,2} \times L^{1,2}\left(W^{+}\right)$be a weak solution of (1.3). Then

$$
\|\phi\|_{L^{\infty}} \leq \max \left\{-\min _{x \in X} s(x), 0\right\}
$$

Proof. We use the method of Taubes [T3] to prove the lemma.

Let $s_{0}=\min _{x \in X} s(x)$. If $s_{0} \geq 0$, then from the first equation of (1.6), (recall that (1.3) and (1.6) are equivalent) we have

$$
\int\left|\nabla_{A} \phi\right|^{2}+\frac{s}{4}|\phi|^{2}+\frac{1}{4}|\phi|^{4}=0
$$

it follows that $\phi=0$. So we may assume $s_{0}=-1$. Define a test section $\eta \in$ $L^{1,2}\left(W^{+}\right)$by

$$
\eta= \begin{cases}(|\phi|-1) \frac{\phi}{|\phi|}, & \text { for }|\phi|>1 \\ 0, & \text { for }|\phi| \leq 1\end{cases}
$$

Let $\nu=\phi /|\phi|$ for $|\phi| \geq 1$. It is clear that $|\nu|=1$ and 
Since $(A, \phi)$ is a weak solution of $(1.6)$, we have

$$
\begin{aligned}
0 & =\int_{\Omega}\left\langle\nabla_{A} \phi, \nabla_{A} \eta\right\rangle+\frac{s}{4}\langle\phi, \eta\rangle+\frac{1}{4}|\phi|^{2}\langle\phi, \eta\rangle \\
& =\int_{\Omega}\left\langle\nabla_{A} \phi, \nabla_{A} \eta\right\rangle+\frac{1}{4}\left(|\phi|^{2}+s\right)(|\phi|-1)|\phi| \\
& \geq \int_{\Omega}\left\langle\nabla_{A}, \nabla_{A} \eta\right\rangle+\frac{1}{4}\left(|\phi|^{2}-1\right)(|\phi|-1)|\phi|,
\end{aligned}
$$

for $s \geq-1$. Here, $\Omega=\{x \in X \| \phi \mid(x)>1\}$. The second term of the last integration is nonnegative. And the first term is also nonnegative. In fact, we have, for $x \in \Omega$,

$$
\begin{aligned}
\left\langle\nabla_{A} \phi, \nabla_{A} \eta\right\rangle & =\left\langle\nabla_{A} \phi, d|\phi| \nu\right\rangle+\left\langle\nabla_{A} \phi,(|\phi|-1) \nabla_{A} \nu\right\rangle \\
& =(|\phi|-1)\left|\nabla_{A} \nu\right|^{2}+\left\langle\nabla_{A} \phi, \nu\right\rangle^{2}+(|\phi|-1)^{2}\left|\nabla_{A} \nu\right|^{2} \\
& +(|\phi|-1)\left\langle\nabla_{A} \phi, \nu\right\rangle\left\langle\nu, \nabla_{A} \nu\right\rangle \\
& \geq \frac{1}{2}\left\langle\nabla_{A} \phi, \nu\right\rangle^{2}+(|\phi|-1)\left|\nabla_{A} \nu\right|^{2} \\
& \geq 0
\end{aligned}
$$

it follows that the set $\Omega$ has measure zero, in other words,

$$
\|\phi\|_{L^{\infty}} \leq 1
$$

In the general case, i.e. without the normaliztion $s_{0}=-1$, the prceding arguments imply

$$
\|\phi\|_{L^{\infty}} \leq \max \left\{-s_{0}, 0\right\}
$$

This completes the proof of the lemma.

The proof of the Theorem 3.1 is now easy:

We are assuming that $(A, \phi)$ is a critical point of $S W$, and thus that $S W(A, \phi)$ is bounded. Lemma 2.5 then implies bounds for $\left\|g^{-1} \phi\right\|_{L^{1,2}}$ and $\|g(A)\|_{L^{1,2}}$. Here we also denote $\left(g(A), g^{-1} \phi\right)$ by $(A, \phi)$.

Next, we have

$$
d^{*} F_{A}=2 d^{*} F_{A}^{+}=-\operatorname{Im}\left\langle\nabla_{i}^{A} \phi, \phi\right\rangle e^{i} \quad(\text { from }(1.6))
$$

Since $\|\phi\|_{L^{\infty}}$ is bounded by Lemma 3.1, this implies

$$
\left\|d^{*} F_{A}\right\|_{L^{2}} \leq c\left(\|\nabla \phi\|_{L^{2}}+\|A\|_{L^{2}}\right)
$$

The second Bianchi identity $d F_{A}=0$ and the ellipticity of $d+d^{*}$ imply

$$
\left\|F_{A}\right\|_{L^{1,2}} \leq c\left(\left\|d^{*} F_{A}\right\|_{L^{2}}+\left\|F_{A}\right\|_{L^{2}}\right)
$$

(3.4), (3.5) and the $L^{1,2}$ estimate for $A$ yield 
and by the Sobolev embedding theorem then also

$$
\|A\|_{L^{r}} \leq c, \quad \text { for any } r<\infty .
$$

From (1.6), we get , for $1<p<2$

$$
\|\Delta \phi\|_{L^{p}} \leq c\left(\left\|\Delta_{A} \phi\right\|_{L^{p}}+\|\nabla A\|_{L^{p}}+\left\|\left|A\left\|\left.\nabla \phi\left|\left\|_{L^{p}}+\right\|\right| A\right|^{2}\right\|_{L^{p}}\right)\right.\right.
$$

and from the Hölder inequality

$$
\left\|\left|a\|\nabla \phi \mid\|_{L^{p}} \leq\|A\|_{L^{\frac{2 r}{2-p}}}\|\nabla \phi\|_{L^{2}}\right.\right.
$$

Thus, by Sobolev's embedding theorem again, $\phi \in L^{1, \frac{4 p}{4-p}}$ and we may then apply the same kind of argument also for $p=2$ and get

$$
\|\phi\|_{L^{2,2}} \leq c
$$

The standard bootstrap argument then gives $L^{k, 2}$ bounds for $(A, \phi)$ for any $k \geq 2$, hence smoothness.

\section{THE COMPACTNESS THEOREM}

Main Theorem. The Seiberg-Witten functional SW satisfies the following PalaisSmale condition:

For any sequence $\left(A_{n}, \phi_{n}\right) \in \mathcal{A}^{1,2} \times L^{1.2}\left(W^{+}\right)$satisfying

(i) $d S W\left(A_{n}, \phi_{n}\right) \rightarrow 0$ strongly in $\mathcal{A}^{-1,2} \times L^{-1,2}\left(W^{+}\right)$;

(ii) $S W\left(A_{n}, \phi_{n}\right) \leq c$, for $n=1,2, \ldots$,

there exists a subsequence (denoted by $\left(A_{n}, \phi_{n}\right)$ ) and $g_{n} \in \mathcal{G}$ such that $g_{n}\left(A_{n}, \phi_{n}\right)$ converges in $\mathcal{A}^{1,2} \times L^{1,2}\left(W^{+}\right)$to a critical point $(A, \phi)$ of $S W$ with $S W(A, \phi)=$ $\lim _{n \rightarrow \infty} S W\left(g_{n}(A, \phi)\right)$.

As we know, the crucial point in the Seiberg-Witten theory and in the preceding arguments is the boundedness of $\|\phi\|_{L^{\infty}}$. But for a Palais-Smale sequence (a sequence satisfying (i) and (ii)), there exists no uniform bound for $\left\|\phi_{n}\right\|_{L^{\infty}}$. This is the main difficulty we encounter here. Fortunately, we can obtain a weaker bound from the proof of (3.2) that suffices for showing the Palais-Smale condition.

\section{Proof of Main Theorem.}

Step 1. By Lemma 2.5, there exist $g \in \mathcal{G}$ with

$$
\left\|g_{n}\left(A_{n}\right)\right\|_{L^{1,2}}+\left\|g^{-1} \phi\right\|_{L^{1,2}} \leq c
$$

(independent of $\mathrm{n}$ ). For simplicity, we denote $g_{n}(A)$ by $A_{n}$, and $g^{-1} \phi_{n}$ by $\phi_{n}$.

From Rellich's Theorem and Sobolev's embedding theorem, there exists a subsequence (also denoted by $\left(A_{n}, \phi_{n}\right)$ ) such that

(i) $A_{n} \rightarrow A$ weakly in $\mathcal{A}^{1,2}$, and $\phi_{n} \rightarrow \phi$ weakly in $L^{1,2}\left(W^{+}\right)$.

(ii) $A_{n} \rightarrow A$ weakly in $\mathcal{A}^{0,4}$, and $\phi_{n} \rightarrow \phi$ weakly in $L^{0,4}\left(W^{+}\right)$. 
Step 2. $(A, \phi)$ is a weak solution of (1.6).

For any 1 -form $\theta \in \mathcal{A}^{1,2}$ (here we abuse the notation a bit),

$$
\int\left\langle F_{A_{n}}, d \theta\right\rangle+\operatorname{Im}\left\langle\nabla_{i}^{A_{n}} \phi_{n}, \phi_{n}\right\rangle\left\langle e^{i}, \theta\right\rangle=d S W\left(A_{n}, \phi_{n}\right)(\theta)=o(1) .
$$

From step 1 (i), we know

$$
\int\left\langle F_{A_{n}}, d \theta\right\rangle=\int\left\langle F_{A}, d \theta\right\rangle+o(1)
$$

Now we show that

$$
\int \operatorname{Im}\left\langle\nabla_{i}^{A_{n}} \phi_{n}, \phi_{n}\right\rangle\left\langle e^{i}, \theta\right\rangle=\int \operatorname{Im}\left\langle\nabla_{i}^{A} \phi, \phi\right\rangle\left\langle e^{i}, \theta\right\rangle+o(1)
$$

This follows from

$$
\begin{aligned}
& \int \operatorname{Im}\left\langle\nabla_{i}^{A_{n}} \phi_{n}, \phi_{n}\right\rangle\left\langle e^{i}, \theta\right\rangle-\int \operatorname{Im}\left\langle\nabla_{i}^{A} \phi, \phi\right\rangle\left\langle e^{i}, \theta\right\rangle \\
& \left.=\int\left\langle e^{i}, \theta\right\rangle\left(\operatorname{Im}\left\langle\nabla_{i} \phi_{n}+A_{n}^{i} \phi_{n}\right), \phi_{n}\right\rangle-\operatorname{Im}\left\langle\nabla_{i} \phi+A^{i} \phi, \phi\right\rangle\right) \\
& =\int\left\langle e^{i}, \theta\right\rangle\left(\operatorname{Im}\left\langle\nabla_{i}\left(\phi_{n}-\phi\right)+A_{n}^{i} \phi_{n}-A^{i} \phi, \phi_{n}\right\rangle-\operatorname{Im}\left\langle\nabla_{i} \phi+A^{i} \phi, \phi-\phi_{n}\right\rangle\right) \\
& =\int\left\langle e^{i}, \theta\right\rangle\left(\operatorname{Im}\left\langle\nabla_{i}\left(\phi_{n}-\phi\right)+A_{n}^{i} \phi-A^{i} \phi, \phi\right\rangle-\operatorname{Im}\left\langle\nabla_{i} \phi_{n}+A^{i} \phi, \phi\right\rangle\right)+o(1)
\end{aligned}
$$

(from step 1 (i) and (iii))

$$
\begin{aligned}
& =\int\left\{\operatorname{Im}\left\langle A_{n}^{i}\left(\phi_{n}-\phi\right), \phi\right\rangle+\operatorname{Im}\left\langle\left(A_{n}^{i}-A^{i}\right) \phi, \phi\right\rangle\right\}\left\langle e_{i}, \theta\right\rangle+o(1) \\
& =o(1) . \quad(\text { from step } 1 \text { (iii) })
\end{aligned}
$$

Here $A-A_{0}=A^{i} e^{i}$ and $A_{n}-A_{0}=A_{n}^{i} e^{i}$. From (4.1)-(4.3), we have for any $\theta$,

$$
\int\left\langle F_{A}, d \theta\right\rangle+\operatorname{Im}\left\langle\nabla_{i}^{A} \phi, \phi\right\rangle\left\langle e^{i}, \theta\right\rangle=0
$$

i.e. $(A, \phi)$ satisfies weakly the second equation of (1.6). Using the same argument, we can show that $(A, \phi)$ satisfies weakly the first equation of $(1.6)$. That is, $(A, \phi)$ is a weak solution of (1.6).

Hence from Theorem 3.1, there exists $g \in \mathcal{G}$ such that $g(A, \phi)$ is a smooth solution of (1.6) and $|\phi(x)| \leq s_{0}$ (recall that $s_{0}=\max \left\{-\min _{x \in X} s(x), 0\right\}$ ). So we may assume that $(A, \phi)$ is a smooth solution.

Step 3. As in the proof of Lemma 3.2, we assume $s_{0}:=\min _{x \in X} s(x)=-1$. Set

$$
\Omega_{n}:=\left\{x \in X|| \phi_{n} \mid>1\right\}
$$


Claim.

$$
\int_{\Omega_{n}}\left|\left\langle\nabla_{A_{n}} \phi_{n}, \nu_{n}\right\rangle\right|^{2} \rightarrow 0, \text { as } n \rightarrow \infty .
$$

As in the proof of lemma 3.2, we have (see (3.2))

$$
\int_{\Omega_{n}}\left|\left\langle\nabla_{A_{n}} \phi_{n}, \nu_{n}\right\rangle\right|^{2} \leq 2 d S W\left(A_{n}, \phi_{n}\right)\left(\eta_{n}\right) \leq 2\left\|d S W\left(A_{n}, \phi_{n}\right)\right\|_{L^{-1,2}}\left\|\eta_{n}\right\|_{L^{1,2}}
$$

Here $\eta_{n}$ is defined as $\eta$ in the proof of Lemma 3.2, namely,

$$
\eta_{n}= \begin{cases}\left(\left|\phi_{n}\right|-1\right) \frac{\phi_{n}}{\left|\phi_{n}\right|}, & \text { for }\left|\phi_{n}\right|>1 \\ 0, & \text { for }\left|\phi_{n}\right| \leq 1\end{cases}
$$

It is sufficient to show that $\left\|\eta_{n}\right\|_{L^{1,2}} \leq c$ for a constant $c$. From the definition of $\eta_{n}$, we have

$$
\left\|\eta_{n}\right\|_{L^{2}}^{2}=\int_{\Omega_{n}}\left(\left|\phi_{n}\right|-1\right)^{2} \leq \int_{X}\left|\phi_{n}\right|^{2}+c \leq\left\|\phi_{n}\right\|_{L^{1,2}}^{2}+c \leq c
$$

and

$$
\begin{aligned}
\left\|\nabla \eta_{n}\right\|_{L^{2}}^{2} & =\left\|d\left|\phi_{n}\right| \nu_{n}+\left(\left|\phi_{n}\right|-1\right) \nabla \nu_{n}\right\|_{L^{2}\left(\Omega_{n}\right)}^{2} \\
& \leq 2\left\|\nabla \phi_{n}\right\|_{L^{2}}^{2}+8\left\|\frac{|\phi|-1}{|\phi|}\right\|_{L^{\infty}\left(\Omega_{n}\right)}\left\|\nabla \phi_{n}\right\|_{L^{2}}^{2} \\
& \leq c\left\|\nabla \phi_{n}\right\|_{L^{2}}^{2} \leq c .
\end{aligned}
$$

Step 4. $A_{n} \rightarrow A$ strongly in $\mathcal{A}^{1,2}$.

From step 1 (i) and (4.1), we have

$$
\begin{aligned}
\left\|F_{A_{n}}-F_{A}\right\|_{L^{2}}^{2} & =\int_{X}\left\langle d\left(A_{n}-A\right), d\left(A_{n}-A\right)\right\rangle \\
& =\int_{X}\left\langle d A_{n}, d\left(A_{n}-A\right)+\int_{X}\left\langle d A, d\left(A_{n}-A\right)\right\rangle\right. \\
& =-\int_{X} \operatorname{Im}\left\langle\nabla_{i}^{A_{n}} \phi_{n}, \phi_{n}\right\rangle\left\langle e^{i}, A_{n}-A\right\rangle+o(1)
\end{aligned}
$$

To use step 3, we decompose $X$ as $\Omega_{n}$ and $X \backslash \Omega_{n}$. On $X \backslash \Omega_{n},\left|\phi_{n}\right| \leq 1$. So it is clear that

$$
\begin{aligned}
& -\int_{X \backslash \Omega_{n}} \operatorname{Im}\left\langle\nabla_{i}^{A_{n}} \phi_{n}, \phi_{n}\right\rangle\left\langle e^{i}, A_{n}-A\right\rangle \\
\leq & \int_{X \backslash \Omega_{n}}\left(\left|\nabla \phi_{n}\right|+\left|A_{n}\right|\right)\left|A_{n}-A\right|^{2} \\
\leq & \left(\left|\left(\left|\nabla \phi_{n}\right|^{2}+\left|A_{n}\right|^{2}\right)^{1 / 2} \int\right| A_{n}-\left.A\right|^{2}\right.
\end{aligned}
$$


On the other hand,

$$
\begin{aligned}
& -\int_{\Omega_{n}} \operatorname{Im}\left\langle\nabla_{i}^{A_{n}} \phi_{n}, \phi_{n}\right\rangle\left\langle e^{i}, A_{n}-A\right\rangle \\
\leq & \int_{\Omega_{n}}\left|\left\langle\nabla_{i}^{A_{n}} \phi_{n}, \nu_{n}\right\rangle\right|\left|\phi_{n} \| A_{n}-A\right| \\
\leq & \left(-\int_{\Omega_{n}}\left|\left\langle\nabla_{i}^{A_{n}}, \nu_{n}\right\rangle\right|^{2}\right)^{1 / 2}\left\|\phi_{n}\right\|_{L^{4}}\left(\left\|A_{n}\right\|_{L^{4}}+\|A\|_{L 4}\right) \\
& \rightarrow 0, \quad \text { as } n \rightarrow \infty \quad \text { (using step 3). }
\end{aligned}
$$

(4.5), (4.6) and (4.7) imply

$$
\left\|A_{n}-A\right\|_{L^{1,2}} \rightarrow 0, \quad \text { as } \quad n \rightarrow \infty .
$$

Step 5. $\phi_{n} \rightarrow \phi$ strongly in $L^{1,2}\left(W^{+}\right)$.

First, from step 1 (i), we have

$$
\begin{aligned}
\left\|\nabla \phi_{n}-\nabla \phi\right\|_{L^{2}}^{2} & =\int_{X}\left(\left\langle\nabla \phi_{n}, \nabla\left(\phi_{n}-\phi\right)\right\rangle+\left\langle\nabla \phi, \nabla\left(\phi_{n}-\phi\right)\right\rangle\right) \\
& =\int_{X}\left\langle\nabla \phi_{n}, \nabla\left(\phi_{n}-\phi\right)\right\rangle+o(1) .
\end{aligned}
$$

$\nabla \phi_{n}=\nabla_{A_{n}} \phi_{n}-A_{n} \phi_{n}$, so we have

$$
\begin{aligned}
& \int_{X}\left\langle\nabla \phi_{n}, \nabla\left(\phi_{n}-\phi\right)\right\rangle \\
= & \int_{X}\left\langle\nabla_{A_{n}} \phi_{n}, \nabla_{A_{n}}\left(\phi_{n}-\phi\right)\right\rangle-\int_{X}\left\langle A_{n} \phi_{n}, \nabla\left(\phi_{n}-\phi\right)\right\rangle \\
& -\int_{X}\left\langle\nabla \phi_{n}, A_{n}\left(\phi_{n}-\phi\right)\right\rangle+\int_{X}\left\langle A_{n} \phi_{n}, A_{n}\left(\phi_{n}-\phi\right)\right\rangle .
\end{aligned}
$$

From (i) of the Palais-Smale condition, we know

$$
\begin{aligned}
& \int_{X}\left\langle\nabla_{A_{n}} \phi_{n}, \nabla_{A_{n}}\left(\phi_{n}-\phi\right)\right\rangle \\
= & -\int_{X} \frac{1}{4}\left(\left|\phi_{n}\right|^{2}+s\right)\left\langle\phi_{n}, \phi_{n}-\phi\right\rangle+d S W\left(A_{n}, \phi_{n}\right)\left(\phi_{n}-\phi\right) \\
= & -\int_{X} \frac{1}{4}\left(\left|\phi_{n}\right|^{2}+s_{0}\right)\left\langle\phi_{n}, \phi_{n}-\phi\right\rangle+\int_{X}\left(s_{0}-s\right)\left\langle\phi_{n}, \phi_{n}-\phi\right\rangle+o(1) \\
= & -\int_{X} \frac{1}{4}\left(\left|\phi_{n}\right|^{2}+s_{0}\right)\left|\phi_{n}-\phi\right|^{2}-\int_{X} \frac{1}{4}\left(\left|\phi_{n}\right|^{2}+s_{0}\right)\left\langle\phi, \phi_{n}-\phi\right\rangle+o(1) \\
& \left(\text { since } \phi_{n} \rightarrow \phi \text { strongly in } L^{2}\right) \\
\leq & -\frac{1}{4}\left(\left|\phi_{n}\right|^{2}+s_{0}\right)\left\langle\phi, \phi_{n}-\phi\right\rangle+o(1) \\
\leq & \frac{1}{4}\left(\left.\int_{X}|| \phi_{n}\right|^{2}+\left.s_{0}\right|^{2}\right)^{1 / 2} \int_{X}\left|\phi_{n}-\phi\right|^{2}+o(1) \\
& \quad\left(\text { since }|\phi| \leq s_{0} \text { by Lemma } 3.1\right)
\end{aligned}
$$


as $n \rightarrow \infty$.

The other three terms can be estimated using step 4. For example, the second term

$$
\begin{aligned}
& \int_{X}\left\langle A_{n} \phi_{n}, \nabla\left(\phi_{n}-\phi\right)\right\rangle \\
= & \int_{X}\left\langle\left(A_{n}-A\right) \phi_{n}, \nabla\left(\phi_{n}-\phi\right)\right\rangle+\left\langle A \phi_{n}, \nabla\left(\phi_{n}-\phi\right)\right\rangle \\
\leq & \left\|A_{n}-A\right\|_{L^{4}}\left\|\phi_{n}\right\|_{L^{4}}\left\|\phi_{n}-\phi\right\|_{L^{1,2}} \\
& +\int_{X}\left\langle A\left(\phi_{n}-\phi\right), \nabla\left(\phi_{n}-\phi\right)\right\rangle+\left\langle A \phi, \nabla\left(\phi_{n}-\phi\right)\right\rangle \\
\leq & \left\|A_{n}-A\right\|_{L^{4}}\left\|\phi_{n}\right\|_{L^{4}}\left\|\phi_{n}-\phi\right\|_{L^{1,2}} \\
& +\left\|\phi_{n}-\phi\right\|_{L^{2}}\left\|\nabla\left(\phi_{n}-\phi\right)\right\|_{L^{2}}+\|A \phi\|_{L^{1,2}}\left\|\phi_{n}-\phi\right\|_{L^{2}} \\
= & o(1)
\end{aligned}
$$

Therefore, we show that $\left\|\phi_{n}-\phi\right\|_{L^{1,2}} \rightarrow 0$ as $n \rightarrow \infty$. Together with step 4 , this completes the proof of the Main Theorem.

\section{REFERENCES}

[DK] S. K. Donaldson and P. B. Kronheimer, The geometry of four-manifolds, Oxford Science Publications, 1990.

[EL] J. Eells and L. Lemaire, Another report on harmonic maps, Bull. London Math. Soc. 20 (1988), 385-524.

[FU] D. Freed and K. K. Uhlenbeck, Instantons and four manifolds, Springer-Verlag New York, 1984.

$[\mathrm{KM}]$ P. B. Kronheimer and T. S. Mrowka, The genus of embedded surfaces in the projective space, Math. Res. Letters 1 (1994), 797-808.

[Le] C. LeBrun, Einstein metrics and Mostow rigidity, Math. Res. Letters 2 (1995), 1-8.

[LM] H. B. Lawson and M.-L. Michelsohn, Spin geometry, Priceton, New Jersey, 1989.

[P] R. S. Palais, Foundations of global non-linear analysis, W. A. Benjamin, Inc. New York, 1968.

[SW1] N. Seiberg and E. Witten, Electromagnetic duality, monopole condensation and confinement in N=2 supersymmetric Yang-Mills theory, Nucl. Phys. B426 (1994), 19-52.

[SW2] N. Seiberg and E. Witten, Monopoles, duality and chiral symmetry breaking in N=2 supersymmetry QCD, Nucl. Phys. B431 (1994), 581-640.

[T1] C. H. Taubes, The Seiberg-Witten invariants and symplectic forms, Math. Res. letters 1 (1994), 809-822.

[T2] - The Seiberg-Witten invariants and the Gromov invariants.

[T3] - On the equivalence of the first and second order equations for gauge theories, Comm. Math. Phys. 75 (1980), 207-227.

[T4] - More constraints on symplectic manifolds from Seiberg-Witten invariants, Math. Res. Letters 2 (1995), 9-14.

[U] K. K. Uhlenbeck, Removable singularities in Yang-Mills fields, Comm. Math. Phys 83 (1982), 31-42.

[W] E. Witten, Monopoles and 4-manifolds, Math. Res. Letters 1 (1994), 769-796.

[Z] J. Zhou, A vanishing theorem for Seiberg-Witten invariants.

Ruhr-Universität Bochum, Fakultät für Mathematik, 44780 Bochum, Germany

E-mail address: juergen.jost@ruba.rz.ruhr-uni-bochum.de

Ruhr-Universität Bochum, Fakultät für Mathematik, 44780 Bochum, Germany

E-mail address: xiaowei.peng@ruba.rz.ruhr-uni-bochum.de

Institute of Systems Science, Academia Sinica, 100080 Beijing, China 